\title{
Frontières
}

\section{Usages politiques de la mort}

\section{Pascal Hintermeyer}

Volume 19, numéro 1, automne 2006

Enjeux politiques et mort

URI : https://id.erudit.org/iderudit/016629ar

DOI : https://doi.org/10.7202/016629ar

Aller au sommaire du numéro

Éditeur(s)

Université du Québec à Montréal

ISSN

1180-3479 (imprimé)

1916-0976 (numérique)

Découvrir la revue

Citer cet article

Hintermeyer, P. (2006). Usages politiques de la mort. Frontières, 19(1), 9-14. https://doi.org/10.7202/016629ar

\section{Résumé de l'article}

La conjonction du politique et de la mort peut être envisagée à partir de la relation anthropologique qui les lie et du mouvement de longue durée par lequel elles tendent à se rapprocher. Avec la modernité, la promotion de la vie apparaît comme un objectif consensuel aboutissant à un traitement systématique de la mort. Trois exemples d'usage politique de la mort sont ici développés. Le premier travaille à l'accroissement de la vie jusqu'à tirer de la mort un surcroît d'existence. Le deuxième, à travers les honneurs rendus aux défunts, illustre des valeurs collectives susceptibles de renforcer la cohésion des vivants. Le troisième utilise la confrontation avec la mort pour renforcer la légitimité de l'action politique. Dans tous ces exemples, le politique retourne à son profit la puissance associée à la mort.
Ce document est protégé par la loi sur le droit d'auteur. L'utilisation des services d'Érudit (y compris la reproduction) est assujettie à sa politique d'utilisation que vous pouvez consulter en ligne.

https://apropos.erudit.org/fr/usagers/politique-dutilisation/ 


\section{Résumé}

La conjonction du politique et de la mort peut être envisagée à partir de la relation anthropologique qui les lie et du mouvement de longue durée par lequel elles tendent à se rapprocher. Avec la modernité, la promotion de la vie apparaît comme un objectif consensuel aboutissant à un traitement systématique de la mort. Trois exemples d'usage politique de la mort sont ici développés. Le premier travaille à l'accroissement de la vie jusqu'à tirer de la mort un surcroît d'existence. Le deuxième, à travers les honneurs rendus aux défunts, illustre des valeurs collectives susceptibles de renforcer la cohésion des vivants. Le troisième utilise la confrontation avec la mort pour renforcer la légitimité de l'action politique. Dans tous ces exemples, le politique retourne à son profit la puissance associée à la mort.

Mots clés: Action politique - honneur mort.

\section{Abstract}

The conjunction of politics and death can be conceptualized from their anthropological foundation and from their longterm developments, which tend to forge a common space. With modernity the promotion of life appears as a universal consensus, culminating in a systematic treatment of death. Three examples of how the political sphere uses death are developed in this paper. The first highlights the emphasis on life to transform death into an added source of existence. The second shows how honoring the dead epitomizes certain collective values thereby reinforcing social cohesion of the living. The third example explores how confrontation with death can be used to reinforce the legitimacy of political action. In each of these cases, the political sphere uses the strength of associations with death to its own benefit.

Keywords: Political action - honordeath.

\section{USAGES POLITIQUES DE LA MORT}

\section{Pascal Hintermeyer, \\ directeur des Sciences sociales, Pratiques sociales et Développement, Université Marc-Bloch, Strasbourg.}

Entre le politique et la mort, il y a une relation anthropologique fondamentale, à la fois originaire, récurrente et renouvelée. D'emblée, la crainte de la mort est une des raisons qui poussent les hommes à s'assembler, à se prêter main-forte et à structurer leur collaboration. Cette visée protectrice favorise les dispositions à l'auto-organisation dans la durée et conduit à prendre en compte la dimension politique. Entre mort et politique, une relation étroite s'établit et s'approfondit, si bien que chacune de ces puissances renvoie à l'autre et se définit de plus en plus par rapport à elle.

Le terme de politique possède de multiples acceptions. Employé au masculin, il suggère un niveau plus essentiel que les affrontements partisans ou l'habileté de responsables qui profitent des circonstances pour faire prévaloir leurs vues et cultivent ce que Condorcet appelait «l'art social». En un sens large, le politique répond à des attentes collectives et recherche les moyens appropriés à la survie et aux intérêts fondamentaux d'une population. Cette préoccupation se prolonge par une conduite délibérée et concertée en vue d'objectifs identifiables.
Une finalité majeure de l'action politique consiste à sauvegarder un ensemble d'êtres humains. Cela signifie d'abord assurer l'existence de la collectivité qu'ils forment: la protéger des agressions extérieures, maintenir sa continuité par la transmission entre les générations, éviter les dissensions internes et tout ce qui peut provoquer un affaiblissement du groupe. L'attitude par rapport à l'existence individuelle dérive de cette volonté de préservation collective : d'une part, la puissance du groupe dépend de la vie de ses membres; d'autre part, la défense de chacun impose de restreindre et de désamorcer la violence interne, y compris en s'en prenant à l'existence de ceux qui refusent de se soumettre à une telle discipline. Les autres menaces contre la vie individuelle étaient traditionnellement tenues pour inéluctables. La mort, chevillée à la condition humaine, échappait pour une large part à la politique.

L'hypothèse que nous nous proposons d'étayer consiste à soutenir qu'avec la modernité, la mort a fait l'objet d'un traitement politique volontariste. Par l'expression «politique de la mort», nous entendons, d'une part, un ensemble d'attitudes systématiquement adoptées dans la société moderne à l'égard de la mort 


\section{LA MODERNITÉ REGORGE DE PHANTASMES}

\section{CONSISTANT À TRANSFORMER LA MORT EN SOURCE DE VIE.}

de ses membres, d'autre part, les prises de position induites par une telle volonté d'organisation et, le cas échéant, leur mise en œuvre par l'État (Hintermeyer, 1981). Nous allons d'abord analyser la conjonction de la politique et de la mort comme l'aboutissement de deux évolutions croisées. Puis nous envisagerons quelques exemples d'usage politique de la mort. Ils illustrent différentes manières de tirer parti de la mort pour affirmer des valeurs collectives et légitimer leur prise en charge politique.

\section{L'APPROFONDISSEMENT DE LA RELATION

ENTRE POLITIQUE ET MORT

L'importance de la dimension politique pour l'actualisation des potentialités humaines a été soulignée dès l'Antiquité. Aristote considérait que la cité était le moyen que les hommes avaient inventé pour tirer parti de leurs diversités et organiser leur collaboration. Ces significations, qui se sont maintenues jusqu'à nos jours, supposaient déjà des garanties pour l'existence individuelle : à l'intérieur de l'espace de la cité, la loi prévaut sur la force et les conflits ne sauraient dégénérer jusqu'au point où ils menaceraient l'existence des protagonistes. À vrai dire, la protection bénéficie pendant l'Antiquité essentiellement à la catégorie juridique des citoyens. Ceux-ci peuvent décider d'accueillir un nouveau-né ou de s'en débarrasser et leurs esclaves ne sont pour eux guère davantage que des «instruments animés» (Aristote, 1961). Malgré ces restrictions, le citoyen antique peut être considéré, selon les termes d'Aristote, comme un "être politique» (Id.). D'abord, parce que, soucieux de vivre et de «bien vivre», il s'intéresse à la chose publique. Ensuite, parce que, réciproquement, la cité s'intéresse à lui et aux manières de l'éduquer afin qu'il assume ses devoirs envers la collectivité (Platon, 1966). La pensée politique de l'Antiquité énonce ainsi, en dépit de ses limitations juridiques et territoriales, des problèmes qui sont toujours au cœur de la réflexion contemporaine.

Lorsque les cités se sont fondues dans des ensembles politiques plus vastes, la vie individuelle est devenue à la fois plus et moins précieuse. Elle s'est avérée plus précieuse parce que la pacification s'est étendue à des aires géographiques beaucoup plus vastes (pax romana puis paix de Dieu). Dans un monde moins cloisonné, «l'étroitesse de l'optique civique» (Veyne, 1976) s’est trouvée dépassée. Avec la dif- fusion du christianisme, le respect pour la vie humaine est devenue une valeur universelle s'appliquant aussi aux esclaves et aux enfants. Mais le changement d'échelle de la conscience humaine s'est aussi traduit par des effets opposés. Les effectifs des États sont devenus considérables et l'attention portée à leurs ressortissants anonymes s'est diluée. De plus, leur autorité s'est trouvée durablement affaiblie au cours de longues époques troublées pendant lesquelles les tence sont ramenées au niveau local. Le seigneur féodal défend ses vassaux, courtisans, paysans et travailleurs en échange de leurs services (Bloch, 1939). Il s'arroge aussi le droit d'éliminer ceux qui portent atteinte à sa souveraineté. C'est seulement peu à peu que la protection de la vie et l'administration de la violence sont assumées, sur toute l'étendue de leur territoire, par les États modernes, qui vont ainsi affirmer leur prépondérance politique et poursuivre les moyens de la consolider (Élias, 1991). Mais, si leur capacité à préserver leurs ressortissants des agressions humaines s'est progressivement accrue, ils restent longtemps encore impuissants à repousser les menaces issues de la nature.

Aux yeux des hommes, la mort représente une préoccupation majeure. Elle est le paradigme de toutes les limitations, le scandale par excellence (Jankélévitch, 1977). Elle était aussi, jusqu'à une époque relativement récente, une puissance familière et largement irréductible à la volonté de l'homme (Ariès, 1977). L'espérance de vie était limitée. L'agriculture occupait la plus grande partie de la population, mais ses techniques étaient rudimentaires et ses rendements faibles. Il suffisait d'une détérioration des conditions climatiques ou de troubles pour compromettre les récoltes et la survie de ceux qui en dépendaient. Le volume des ressources était peu extensible. Toute période favorable, qui avait permis un accroissement de la population, était suivie d'une brutale correction. Les organismes, affaiblis par la malnutrition, résistaient mal aux agents infectieux et nombreux sont ceux qui succombaient à la prochaine épidémie. La mortalité infantile était élevée. Dans un tel contexte, la vie était quelque chose de trop aléatoire pour pouvoir être considérée comme une richesse. Les ravages de la mort semblaient difficiles à éviter, mais ils étaient de plus en plus mal supportés.

La situation se modifie au XVIII ${ }^{\mathrm{e}}$ siècle, grâce à une pluralité d'améliorations dont capacités d'assurer la sécurité de l'exis- résulte un changement dans des proportions sans précédent depuis le Néolithique. L'accroissement des moyens de transport, qui permettent de secourir les régions sinistrées, se conjugue à la "révolution agricole» et à une hygiène moins défectueuse pour autoriser un accroissement de la population au-delà des seuils qui l'avaient auparavant limitée. Et l'espoir se fait jour que de semblables avancées ne soient que le prélude à un développement toujours croissant de l'existence humaine, quantitativement et qualitativement. La célébration de la vie se prolonge en celle des lumières, du bonheur et du progrès. Elle suscite une volonté d'action suivie et concertée, elle débouche sur une attente au plan politique.

Ainsi viennent converger deux mouvements de longue durée. L'un est caractérisé par une extension du domaine politique, dont les moyens et les compétences ne cessent de s'accroître et qui cherche à la fois à assumer plus complètement sa tâche de protection de la vie et à mieux tirer parti des ressources humaines (Foucault, 1976). L'autre tendance est celle d'un reflux de la mort, dont la place dans la conscience et la société moderne se trouve remise en question. À la jonction de ces deux évolutions, le politique investit plus radicalement le domaine de la mort.

\section{LA PROMOTION POLITIQUE ET SCIENTIFIQUE DE LA VIE HUMAINE}

Nous allons à présent nous attacher à trois exemples d'usage politique de la mort. Ils témoignent d'une même inspiration, celle de retourner la puissance de la mort et de s'en servir. Le premier se situe aux frontières de la vie et de la mort. Celles-ci se trouvent délibérément rectifiées au détriment de cette dernière. Avec la modernité, les fondements traditionnels de l'ordre social sont remis en cause. Les valeurs susceptibles de susciter une adhésion générale en prennent d'autant plus d'importance. Parmi ces références communes, la promotion de la vie humaine fait consensus. Elle peut servir de justification à l'action politique et à ceux qui s'y vouent. En deçà des péripéties auxquelles ils sont confrontés, leur tâche se définit par la volonté d'étendre l'empire de la vie humaine, notamment son allongement, en assurant les conditions permettant de profiter des avancées scientifiques et thérapeutiques. Là où une telle évolution se heurte à ses limites, une deuxième ressource consiste à affirmer une continuité entre la vie et la mort en rendant hommage aux défunts pour promouvoir des valeurs collectives. Le troisième exemple illustrera la manière dont la confrontation avec la mort peut être utilisée pour renforcer la légitimité de l'homme politique. 


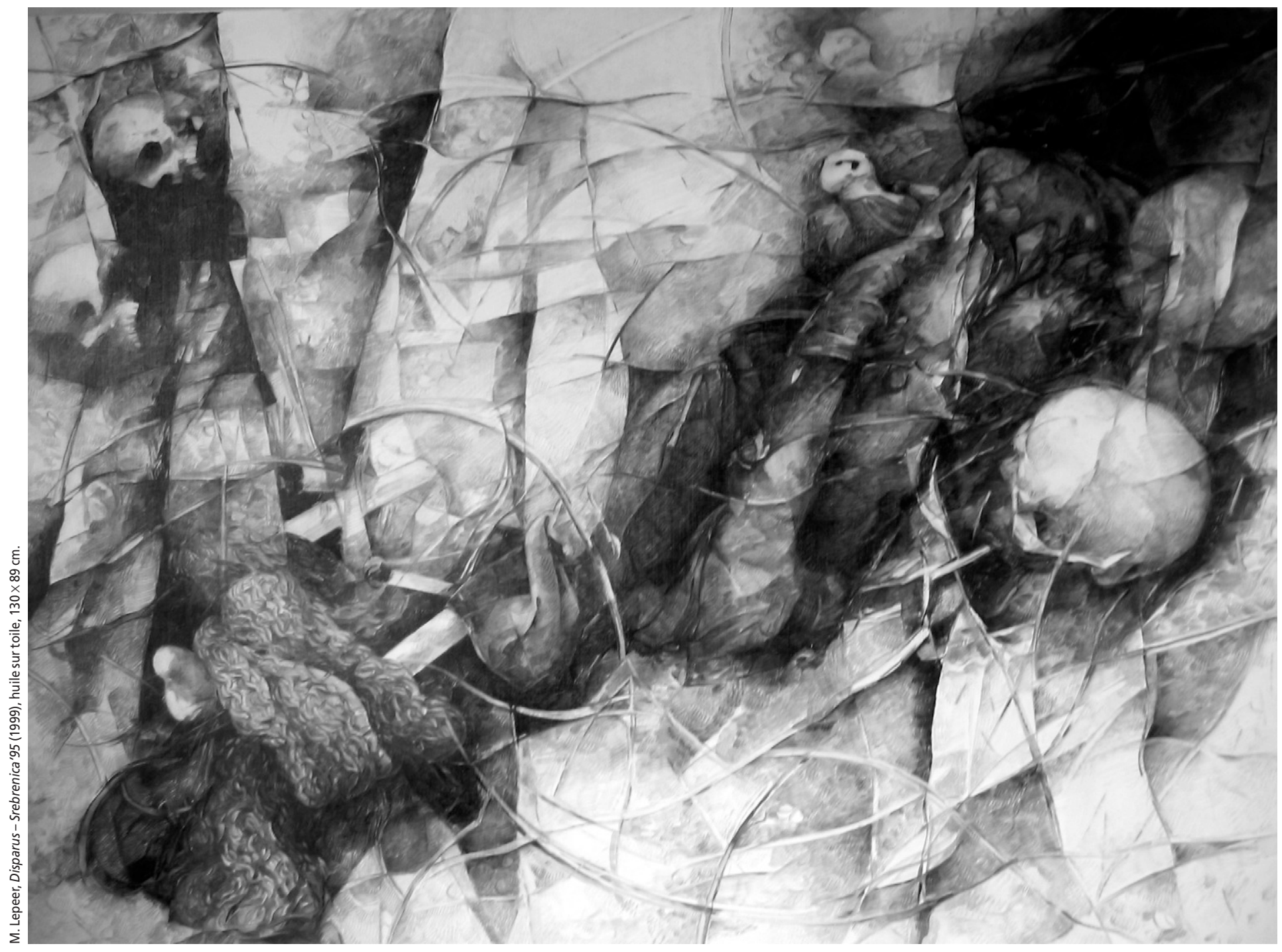

L'accroissement de la vie résulte pour une part de la capacité à tirer parti de son contraire. Le sacrifice représente une tentative pour inverser la destruction en fécondité (Morin, 1970). La modernité regorge de phantasmes consistant à transformer la mort en source de vie. Dès le $\mathrm{XVIII}^{\mathrm{e}}$ siècle, on cherchait à tirer parti de la matérialité des cadavres, par exemple en utilisant leur décomposition comme engrais organique propice aux rendements agricoles. L'utopie utilitariste est aujourd'hui étayée et dépassée par les techniques médicales de pointe. Le corps en fin de vie est devenu une réserve potentielle d'organes à recycler. En France, les obstacles ont été partiellement levés par l'adoption le 22 décembre 1976 de la loi Caillavet, qui postule que la personne décédée consent à l'utilisation de son corps, à moins qu'elle ne s'y soit opposée de son vivant. Les médecins, ainsi juridiquement couverts, s'enquièrent tout de même en pratique des avis que le défunt a pu communiquer à ce sujet à ses proches. Mais ceux-ci ne sont souvent que partiellement informés de l'étendue des prélèvements effectués. Quelque 20000 personnes vivent actuellement en France avec un organe transplanté et 5000 sont en attente d'une greffe de ce type.

La pénurie d'organes disponibles, l'obligation d'intervenir rapidement, la nécessité de s'assurer au préalable de la compatibilité entre donneur et receveur ainsi que de l'absence de risque de contamination entre eux requièrent des procédures de gestion performantes, fiables et rapides. Celles-ci permettent de saisir l'occasion, souvent consécutive à une mort violente. Elles transforment cet événement tragique en opportunité de sauver une vie. La mort est alors à l'origine d'un supplément d'existence. Et celui qui fait don de ses organes peut espérer ne pas mourir tout à fait puisqu'il survit partiellement dans un ou plusieurs autres corps qui lui resteront éternellement débiteurs. Une condition de telles prouesses est évidemment la possibilité de maintenir en vie les organismes sur lesquels sont effectués les prélèvements. Il faut aussi que de telles opérations ne puissent être assimilées à un homicide. Cette double contrainte explique pour une part la modification des critères juridiques de la mort à la fin des années 1960, les dispositions alors adoptées sur la mort cérébrale ayant notamment pour but de faciliter les greffes. Cette ambition suppose aussi la possibilité de contrôler et de retarder le passage vers la mort. Cela incline à penser que la définition actuellement en vigueur de la mort résulte à la fois de l'intention d'en protéger l'individu menacé et du projet de préserver d'autres vies par le développement des transplantations. Ces objectifs se révèlent ainsi complémentaires. Orientés par la volonté de défendre la vie humaine, ils se rapprochent aujourd'hui de l'idéal d'une gestion optimale des corps.

Parallèlement aux transplantations d'organes, qui se banalisent, de multiples prélèvements, moins spectaculaires mais plus massifs, sont effectués sur des cadavres. Ceux-ci sont devenus le lieu d'activités de récupération multiples qui font des corps morts une source de vie et de richesse. Aux prélèvements osseux 
et transplantations de cornée s'ajoute l'exploitation d'articulations, de ligaments, de valves cardiaques, de vaisseaux (artères et veines), de tendons, de cartilages et de nerfs. La mort fait du corps un magasin remarquablement bien pourvu. Il recèle non seulement des parties nobles et convoitées, ces organes susceptibles d'une nouvelle connexion sur des vies chancelantes qui s'en trouveront consolidées, mais aussi quantité de matériaux, d'accessoires et de composants qui font l'objet d'utilisations diverses. Certes, les réticences des familles freinent encore souvent ces possibilités. Mais les spécialistes avertissent que des restrictions excessives ne feraient que déplacer le problème en direction de pays prêts à faire fi de nos délicatesses pour s'imposer dans ces nouvelles activités et satisfaire une demande croissante.

Les perspectives de prolongation de la vie par recyclage d'éléments de corps morts apparaissent comme un avatar de vieux rêves. On se souvient qu'Esculape avait essayé de ressusciter les morts et en avait été châtié par Zeus. La modernité espère que la technique lui permettra de faire du vivant avec le mort. En 1818, M. Shelley imagine que le docteur Frankenstein crée un être vivant avec des morceaux de cadavres. Si de tels phantasmes peuvent aujourd'hui trouver un début de réalisation, c'est qu'ils ne se bornent pas au délire d'esprits isolés ou de savants fous et à l'écho qu'ils rencontrent dans le public. Les prouesses de la médecine sont sous-tendues par une volonté collective tenace de faire reculer la mort.

L'importance de l'engagement politique en faveur du développement de la vie se vérifie aussi en creux à travers les réactions collectives aux situations où les autorités publiques sont mises en cause pour avoir failli à cette mission. En France, le scandale du sang contaminé par le virus du sida ou la surmortalité consécutive à la canicule de 2003 confirment que toute négligence de ce type est politiquement sanctionnée. Plus généralement, le ressort de nombreux mouvements sociaux est la dénonciation d'une perspective mortifère, même métaphorique. Des catégories socioprofessionnelles protestent avec véhémence contre des décisions accusées de conduire ou de consentir à leur disparition. Des restrictions budgétaires sont dénoncées comme attentatoires aux activités qu'elles subventionnaient. Des manifestations écologistes alertent sur la détérioration possible de diverses composantes du milieu naturel. La sensibilité aux dérèglements et aux destructions de l'écosystème témoigne que l'exigence de protection de la vie continue à susciter l'engagement politique tout en revêtant aujourd'hui une signification élargie qui inclut la préservation de l'environnement et du patrimoine constitué par la diversité biologique et culturelle. Devant toute menace potentielle, l'anticipation est de rigueur et le principe de précaution devient un impératif politique catégorique.

\section{L'AFFIRMATION \\ DES VALEURS COLLECTIVES}

La défense de la vie apparaît comme une valeur nodale de la modernité et de l'initiative politique, avec de multiples conséquences. Elle a ainsi été identifiée comme au fondement de l'avènement du droit social (Ewald, 1986). Elle anime aussi les obsessions sécuritaires, l'idéal de réduction des risques et même la rhétorique des «dommages collatéraux » et du « zéro mort » dans la guerre contemporaine (Hintermeyer et Schmoll, 2006). Mais, en dépit de la sollicitude des pouvoirs publics, de la compétence des spécialistes et des rêves d'immortalité, la mort reste l'horizon de la condition humaine. Les hommes cherchent donc à compenser sa venue par un surcroît de cohésion et l'affirmation de valeurs collectives (Durkheim, 1912). Lorsqu'un décès survient, les survivants cherchent à maintenir des continuités. Ces réactions défensives se prolongent en volonté de rechercher jusque dans la mort des éléments propices au développement et à l'amélioration de la vie. Les honneurs rendus aux défunts (étymologiquement ceux qui sont dépourvus de fonction) leur attribuent alors une utilité nouvelle. L'évocation des résultats de leurs entreprises ne se limite pas à l'intérêt de ces dernières, elle illustre des qualités, des attitudes et des valeurs socialement reconnues. Les morts transformés en exemples servent, à travers la reconnaissance de leur contribution au développement collectif, à promouvoir des vertus proposées à l'édification des vivants.

La société, remarquait Auguste Comte, est composée de plus de morts que de vivants. En conservant, en retenant et en répétant leur nom, elle affirme une continuité selon deux modalités complémentaires qui sont aussi deux manières d'euphémiser la mort. La première est la référence à une lignée dont le nom se perpétue à travers le renouvellement des générations. La seconde consiste à préserver et à entretenir la mémoire d'individualités reconnues comme exemplaires. Ce processus de consécration est particulièrement significatif car l'honneur accordé à certains défunts est lié au culte des talents, des dispositions et des qualités qu'ils sont censés avoir incarnés et qui se trouvent ainsi réactivés dans la vie quotidienne. Celle-ci est saturée de noms qui, par la référence aux morts qui les ont portés, lui donnent un surcroît de sens. Le monde contemporain est ainsi rempli de noms de morts plus ou moins illustres.
Les institutions se réfèrent à ceux qui les ont servies pour marquer leur identité et la continuité de leur action par-delà la disparition des individus. Elles cultivent leurs spécificités avec une détermination renforcée par leur attachement à la mémoire et à l'engagement de leurs membres décédés. Aux successeurs de ceux-ci peut incomber la tâche de les honorer, comme dans ces discours de réception à l'Académie française où le nouvel élu glorifie la vie de celui dont il va occuper le fauteuil. L'analyse des éloges prononcés montre que les institutions sélectionnent, dans l'existence du défunt, les événements qui leur ont été bénéfiques ou qui prennent une signification par rapport à elles. L'évocation de la vie donne lieu à sa stylisation sous la forme d'une carrière, selon un schéma ascensionnel appliqué à la fois aux vivants et aux morts.

La consécration sociale est officiellement accordée à divers hommes illustres. Beaucoup de morts, plus ou moins renommés, peuplent ainsi la culture, la cité et la mémoire. Leur nom est attribué aux rues, places, parcs, établissements d'enseignement, de sport et de loisirs, stades et salles, stations et moyens de transport en commun. Il est reproduit sur les pièces de monnaie, les timbres ou les billets de banque. Ces associations entre une personnalité et un lieu ou un objet public ont une portée symbolique. Elles sont à la fois un honneur pour la première et un repère pour les hommes qui fréquentent ou utilisent le second. Ainsi de nombreux morts nous guident dans la vie quotidienne. Leur présence emblématique s'avère d'autant plus précieuse que le présent paraît plus incertain. Elle se manifeste par d'autres signes encore. Les statues de nos grands ancêtres ornent les squares, les demeures qu'ils ont habitées sont signalées, leurs œuvres figurent dans les bibliothèques, leurs portraits occupent les musées et les livres scolaires, où leurs actions mémorables sont racontées et commentées. Leurs principaux anniversaires offrent l'occasion de revisiter leur vie et leurs réalisations. Les commémorations se succèdent et se multiplient. Leur portée politique tient à l'unanimité et à la cohésion qu'elles induisent et qui fait contrepoint aux heurts, désaccords et rivalités entre contemporains. Les hommes dont l'existence est reconnue comme présentant une valeur éminente pour la société sont récompensés par leur présence nominative dans l'espace public et la mémoire collective.

La relation entre un nom propre et une chose peut aussi être plus essentielle. Le droit de donner un nom surtout si c'est le sien représente un honneur insigne, l'acceptation par tous les locuteurs d'une relation intime entre celui qui nomme et la réalité qui se trouve ainsi désignée. Le 
LORSQU'UN DÉCÈS SURVIENT, LES SURVIVANTS CHERCHENT À MAINTENIR DES CONTINUITÉS. CES RÉACTIONS DÉFENSIVES SE PROLONGENT EN VOLONTÉ DE RECHERCHER JUSQUE DANS LA MORT DES ÉLÉMENTS PROPICES AU DÉVELOPPEMENT

ET À L'AMÉLIORATION DE LA VIE.

premier se voit reconnaître un ascendant définitif sur la seconde. Il en est considéré comme le créateur puisque, avant son intervention, elle demeurait innommée. La possibilité de lui attribuer le nom d'un être humain solennise la reconnaissance sociale du caractère fondateur d'une initiative personnelle d'utilité publique. Sous l'Antiquité déjà, les grands conquérants fondaient des villes dont certaines portent encore leur nom. Au $\mathrm{XX}^{\mathrm{e}}$ siècle, des prophètes de la société idéale (Marx, Lénine, Staline) se sont vu décerner pareil honneur. Le continent américain et plusieurs de ses parties sont désignés en référence à des hommes ayant contribué à les découvrir. Les inventions et procédés techniques portent le nom de leur concepteur, les entreprises et les marques de fabrication, celui de leur fondateur. Le même usage s'applique aux axiomes, lois, équations, courbes, principes, théories, dispositions législatives ou réglementaires, maladies, plantes et fleurs. Il en va même ainsi pour diverses commodités, par exemple les poubelles, immortalisant le nom d'un préfet de la Seine, ou les vespasiennes, celui d'un empereur romain. Dans bien des activités sociales, la référence aux morts qui les ont favorisées vaut reconnaissance de dette et affirmation de valeurs partagées.

\section{LA MORT COMME LÉGITIMATION DE L'AUTORITÉ POLITIQUE}

L'homme politique aspire à imprimer sa marque dans la grande histoire et à être révéré par les générations futures. Mais il cherche d'abord à établir son ascendant sur ses concitoyens. La proximité par rapport à la mort constitue un moyen souverain pour y parvenir. Elle concourt à produire et à mettre en évidence des personnalités éminentes aptes à l'exercice des fonctions dirigeantes. La mort est en effet propice à la maîtrise, à la fois maîtrise de soi et maîtrise sur autrui. Elle place le sujet dans la perspective de sa propre disparition et modifie ainsi son point de vue et ses attitudes par rapport à lui-même et par rapport au monde. En regardant la mort en face, il se hisse au-dessus de sa propre personne et s'affranchit des limitations qui affectent le commun des mortels. Il ne se laisse pas abuser par ce que d'ordinaire les hommes redoutent et recherchent. Il n'est pas dupe des passions qui les agitent, des rivalités qui les divisent, des richesses qui les corrompent. Il transcende la faiblesse de son corps, lui impose la force de sa volonté et ne se soumet même pas à l'instinct le plus puissant, celui de sa propre conservation. Il se rend ainsi apte à tenir les rênes de tous les désirs humains, à les brider et à les orienter dans la direction opportune. Il peut aussi opposer à l'adversité une inébranlable fermeté et conserver en toute circonstance courage, sang-froid et contrôle de soi. Il est même réputé savoir relativiser ses propres intérêts. La fréquentation de la mort le rend ainsi supérieur à ce qu'il était et à ceux qui en sont restés éloignés.

La domination charismatique résulte de l'aura que diffuse une personnalité exceptionnelle (Weber, 1971). Celle-ci doit son ascendant non seulement à ses qualités éminentes mais surtout à sa dimension surnaturelle. Elle est en effet réputée entretenir une relation privilégiée, étroite et constante avec une puissance transcendante, invisible et mystérieuse qui demeure d'ordinaire inaccessible aux autres hommes. L'individu qui occupe cette position hors du commun en tire une capacité à effectuer des prouesses et des prodiges qui suscitent l'étonnement, l'admiration, le respect, la ferveur. Il est sollicité pour des conseils, suivi dans ses avis et même dans ses ordres, en somme considéré comme une autorité. Nombreux sont ceux qui sont disposés à s'en remettre à lui dans les décisions les plus importantes de leur existence, à lui confier la direction des affaires individuelles et collectives, à l'accepter pour chef (Brown, 1985). Une telle confiance exige en retour de celui auquel elle est accordée la manifestation de ses dons extraordinaires lors d'épreuves effectives ou rituelles. Les premiers pharaons déjà devaient accomplir pendant leur jubilé à Saqqarah des performances qui témoignaient de la perpétuation de leurs facultés exceptionnelles.

Le principe charismatique n'a pas disparu avec la rationalisation moderne de la société. Au contraire, l'un des dirigeants qui a le plus efficacement œuvré à celle-ci est en général tenu, à juste titre, pour une incarnation typique de celui-là. Napoléon a notamment fondé son pouvoir sur l'intrépidité avec laquelle il affrontait la mort. Il la met en relief, tout au long de sa carrière, en forgeant sa propre légende. Cet exemple montre que la domination charismatique conserve de l'importance dans la modernité et qu'elle est susceptible d'être associée à d'autres et notamment à la domination rationnelle légale. Un dirigeant peut œuvrer à l'élaboration de codes de lois et à la mise en place d'une administration centralisée tout en cherchant à entretenir ou à raviver son prestige grâce à la mort. L'efficacité symbolique de cette dernière est d'ailleurs utilisée jusque dans la défaite et l'exil, comme un moyen de rebondir, voire, lorsque c'est devenu décidément impossible, de marquer encore les esprits et de les préparer à un retournement post mortem. Mais cette épreuve n'a plus tout à fait le sens qu'elle revêtait dans l'Antiquité. Elle n'est plus tant la preuve d'être favorisé par la fortune ou de pouvoir compter sur des forces surnaturelles qu'un gage de cohérence et de fermeté. L'issue du combat importe moins que la détermination à le livrer. On assiste ainsi à une psychologisation du charisme. Cette évolution accorde à la mort une place encore plus grande puisque celle-ci révèle la véritable trempe d'une personnalité. Elle correspond à une situation où le rapport à la mort est devenu moins familier. Le seul fait de l'assumer contribue déjà à distinguer un individu de ses semblables et à lui prêter des qualités qui sortent de l'ordinaire.

Les principaux dirigeants politiques de notre temps entretiennent avec la mort une relation étroite qui apparaît comme l'une des sources de leur légitimité. Ainsi Charles de Gaulle, c'est avant tout l'homme du 18 juin, celui qui refuse de se soumettre quand tout semble perdu, celui qui appelle à continuer le combat, même lorsqu'il paraît sans espoir. C'est aussi une personnalité qui, arrivée au faîte du pouvoir, fait la preuve de sa capacité à y renoncer. Le thème de la traversée du désert, qui est fréquemment évoqué pour décrire sa position sous la IVe République, est d'ailleurs devenu une sorte de passage obligé avant d'accéder au sommet de l'État. Cette épreuve fait penser aux vexations que certains peuples, par exemple les Ndembu de Zambie, imposent à leur prochain souverain (Turner, 1990). Dans ces cas, l'ensemble de la communauté se livre à un rituel d'injures au futur chef. Dans nos sociétés policées, l'aspirant au pouvoir est plutôt confronté à l'échec, à l'indifférence et au désintérêt.

François Mitterrand lui aussi a connu les revers électoraux successifs, les relations difficiles avec ses partenaires de l'union de la gauche, avant d'accéder au pouvoir suprême. Lors de la cérémonie d'investiture 
qui a suivi l'élection présidentielle, il a ajouté à la consécration par les urnes la légitimité par la mort. L'une des étapes les plus significatives de la journée a en effet été celle du Panthéon. Là, il s'est dérobé à la multitude des participants et à l'effervescence de la cité pour se retirer, seul vivant parmi les grands hommes morts, et rester de longs moments en leur unique compagnie. Il suggérait par là une continuité et une connivence avec des personnages illustres dont la mort a ciselé les traits jusqu'à les transmuter en valeur. Il recherchait sans doute aussi un lien avec des puissances qui surplombent la condition humaine, pour saisir «toujours le même phénomène: une sorte de soudaine communication avec ce qu'on peut appeler les espaces sidéraux, qui n'est peut-être tout simplement que le fil rétabli entre le petit individu passager que je suis et les grandes explications permanentes, dont je n'ai pas la clé » (Mitterrand, 1981, p. 19). Cette relation retrouvée rattache l'action actuelle à des fondements intemporels, elle en aiguise le sens et en majore la portée.

Mitterrand fait valoir que la proximité de la mort renforce l'aptitude à mesurer, à évaluer, à remettre les choses, les événements et les hommes à leur juste place. «Présente à l'esprit, la mort est la règle à calcul de la relativité»(Ibid., p. 22). Elle est donc un outil de vérité, un instrument de précision dont le maniement a en retour un effet sur celui qui l'utilise. Elle peut le rendre plus à même de définir ce qu'il est, de saisir ce en quoi consiste sa spécificité. «La certitude de la mort devrait approfondir la conscience de soi-même» (ibid.). Rien d'étonnant alors à ce que la mort, susceptible de tant d'usages politiques, ait joué un rôle aussi important dans la vie politique des années qui ont suivi. À la fin du second septennat, elle a d'ailleurs révélé une autre propriété, de nature protectrice: devant un président qui consacrait à lutter contre la maladie une part manifestement croissante de son énergie, les coups des adversaires se faisaient moins assurés, moins acérés, moins percutants. Peu d'hommes politiques étaient prêts à prendre le risque de «tirer sur l'ambulance » et de contribuer à abattre une personnalité physiquement affaiblie qui donnait l'impression de tirer de cette ultime épreuve un surcroît de sagesse, une capacité à assumer son rôle d'arbitre avec davantage d'élévation et d'impartialité.

Lorsque l'homme politique ne va pas au-devant de la mort, il doit s'attendre à être rattrapé par elle. La vie politique est remplie de défis, de règlements de comptes et de paris périlleux. Elle comporte une dimension irrationnelle qui subsiste même dans les périodes de consensus et celles qui sont censées se conformer à la pensée unique. Elle ressemble à une arène dans laquelle les protagonistes ne cessent de se porter des coups pour tuer symboliquement leurs adversaires sous les yeux de spectateurs qui participent au jeu de massacre par la médiation des journalistes qui leur donnent des nouvelles des affrontements, les commentent en leur nom et dressent des obstacles déstabilisateurs. Pour l'homme politique qui a su éviter les embûches, "rebondir » après avoir été jeté à terre, accéder au pouvoir, «l'épreuve du feu » représente le moment de vérité où se trouve confirmée ou infirmée son aptitude à faire face à la mort. Elle représente pour lui à la fois l'opportunité de constituer «l'union sacrée» autour de sa personne et celle de faire la preuve qu'il est à la hauteur du péril. En tenant bon dans les combats effectifs ou potentiels, il tente de gagner ses galons d'homme d'État.

Entre le politique et la mort se nouent ainsi des relations étroites, sans cesse réactivées, souvent paradoxales. Le sens de l'action politique est en grande partie liée à sa capacité à repousser la mort. Cela suppose d'aller à sa rencontre et de l'affronter afin de retirer des succès obtenus un surcroît de prestige et de légitimité. Les rapports avec cette puissance mystérieuse, effrayante et fascinante à la fois, que les anthropologues appellent, à la suite des travaux publiés par Rudolf Otto pendant la Première Guerre mondiale, le numineux, sont ambivalents. Ils consistent à s'en écarter, mais aussi à s'en servir, notamment par des rites établissant une communication propitiatoire avec les morts (Otto, 1949). Déjà sous ses formes archaïques, le pouvoir cherchait à tirer parti de la mort. Il a ultérieurement persisté dans cette visée tout en diversifiant les moyens d'y accéder. Nous en avons envisagé quelquesuns, qui illustrent certains usages politiques de la mort. Ils consistent à retourner à leur profit la puissance de la mort, à transformer le négatif en source d'affirmation et de cohésion. D'autres exemples pourraient confirmer l'importance renouvelée des relations entre mort et politique. L'effondrement du monde communiste nous a privés à la fin du $\mathrm{XX}^{\mathrm{e}}$ siècle de l'ennemi dans lequel nous nous étions accoutumés à voir une menace pour notre survie et celle de la planète. Le terrorisme n'a pas tardé à nous en fournir un autre et à nous rappeler que la puissance de la mort peut rejaillir sur ceux qui vont à sa rencontre et leur permettre de compenser un rapport de force déséquilibré. Ce défi potentialisé se traduit aujourd'hui par une réactivation des usages politiques de la mort.

\section{Bibliographie}

ARIÈS, P. (1977). L'homme devant la mort, Paris, Seuil.

ARISTOTE (1961). Politique, trad. Tricot, Paris, Vrin.

BAUDRILLARD, J. (1976). L'échange symbolique et la mort, Paris, Gallimard.

BLOCH, M. (1939). La société féodale, Paris, Albin Michel.

BROWN, P. (1985). La société et le sacré dans l'Antiquité tardive, Paris, Seuil.

DURKHEIM, E. (1912). Les formes élémentaires de la vie religieuse, Paris, Alcan.

ÉLIAS, N. (1991). La société des individus, Paris, Fayard.

EWALD, F. (1986). L'État providence, Paris, Grasset.

FOUCAULT, M. (1976). La volonté de savoir, Paris, Gallimard.

HINTERMEYER, P. (1981). Politiques de la mort, Paris, Payot.

HINTERMEYER, P. et P. SCHMOLL (dir.) (2006). Les nouvelles figures de la guerre, Revue des sciences sociales, Strasbourg, Université Marc-Bloch, ${ }^{\circ} 35$.

JANKÉLÉVITCH, V. (1977). La mort, Paris, Flammarion.

MITTERRAND, F. (1981). Politique II, Paris, Fayard.

MORIN, E. (1970). L'homme et la mort, Paris, Seuil, éd. augmentée.

OTTO, R. (1949). Le sacré, Paris, Payot.

PLATON (1966). La République, Paris, Garnier.

THOMAS, L.V. (1978). Mort et pouvoir, Paris, Payot.

TURNER, V.(1990). Le phénomène rituel. Structure et contre-structure, Paris, PUF.

VEYNE, P. (1976). Le Pain et le Cirque: sociologie historique d'un pluralisme politique, Paris, Seuil.

WEBER, M. (1971). Économie et société, Paris, Plon. 